\title{
HLA class II SNP interactions and the association with type 1 diabetes mellitus in Bengali speaking patients of Eastern India
}

\author{
Oindrila Raha ${ }^{1}$, Biswanath Sarkar ${ }^{1}$, Bhaskar VKS Lakkakula², Veerraju Pasumarthy ${ }^{3}$, Sudhakar Godi \\ Subhankar Chowdhury ${ }^{4}$, Pradip Raychaudhuri ${ }^{5}$ and Raghavendra Rao Vadlamudi ${ }^{*}$
}

\begin{abstract}
Background: Several studies have demonstrated a fundamental role for the HLA in the susceptibility of, or protection to, type 1 diabetes mellitus (T1DM). However, this has not been adequately studied in Asian Indian populations. To assess the frequency of HLA class II (DPA1, DPB1, DQA1, DQB1 and DRB1) associated to susceptibility or protection toT1DM in a Bengali population of India with diabetes.

Results: Single nucleotide polymorphism study. The HLA genotyping was performed by a polymerase chain reaction followed by their HLA-DP, DQ, and DRB1 genotypes and haplotypes by sequencing method. The results are studied by Plink software. The $x^{2}$ tests were used for the inferential statistics. To our knowledge, this study is the first of a kind which has attempted to check the HLA association with T1DM by SNPs analysis. The study recruited 151 patients with T1DM and same number of ethno-linguistic, sex matched non-diabetic controls. The present study found a significant SNP rs7990 of HLA-DQA1 ( $p=0.009)$ negative correlation, again indicating that risk from HLA is considerably more with T1DM.
\end{abstract}

Conclusions: This study demonstrates that the HLA class-II alleles play a major role in genetic basis of T1DM.

Keywords: HLA, T1DM, SNP, India, Haplotyping, Alleles

\section{Background}

Type 1 diabetes mellitus (T1DM) (OMIM-222100), results from a cellular-mediated autoimmune destruction of the Beta-cell of the pancreas [1]. T1DM is a disease of major public health concern [2-4]. The previous studies showed that in India, the prevalence of T1DM varies from about 1.6 to 10.5/100000/year [5,6]. The epidemiological study conducted in South Indian population for four years, suggested the prevalence of T1DM in India is increasing. The overall prevalence of T1DM in Karnal district, a North Indian city with a population of 222017, is 10.20/100,000 population [7].

The genetic risk factors of T1DM are better understood than the environmental risk factors. Studies on Human and animal models show the MHC class II-

\footnotetext{
* Correspondence: ansidiabetes@gmail.com

${ }^{6}$ Department of Anthropology, University of Delhi, North Campus, Delhi 110 007, India

Full list of author information is available at the end of the article
}

mediated effects on the disease susceptibility [8]. Early studies identified in the Human Leukocyte Antigen (HLA) genes, located on chromosome 6p21.31 as T1DM susceptibility genes. Resulting studies showed an association between the insulin gene on chromosome 11p15.5. The risk of T1DM is linked with about 18 regions of the genome. These regions, each of which may contain multiple genes, labeled IDDM1 to IDDM18. The best studied is IDDM1, which contains the HLA genes encode proteins of the immune response $[9,10]$.

HLA is one of the most polymorphic genetic systems in Human genome. IMGT/HLA database have reported 6,275 HLA alleles [11]. Because the HLA class II molecules are polymorphic, they can embrace a wide variety of antigens in their antigen-binding groove and present them to diverse T-lymphocyte antigen receptors, triggering antigen recognition. Several studies have displayed HLA class II alleles, DQ and DR influence T1DM susceptibility. The contribution of the DQ

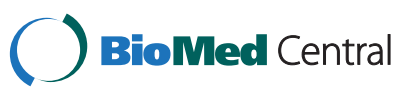


molecules to overall disease susceptibility might be genotype dependent and/or may be influenced by the DRB1*04 allele on the haplotype [12].

The HLA-DQ heterodimers encoded by the DQA1*0301, DQB1*0302 and DQA1*0501, DQB1*0201 alleles have the strongest association with T1DM [13]. These alleles are in LD with the HLA-DR4 and -DR3 alleles. In T1DM at least one allele of DR3 or DR4 is found in 95\% in Europeans, and individuals with both DR3 and DR4 are particularly susceptible to T1DM, whereas, the DR2 allele is protective [14-16].

In North Indian T1DM patients also, the homozygosity and heterozygosity of DRB1*0301 and DRB1*04 alleles is significantly associated [17-19]. The Indian samples show HLA-C*0702 allele and shared HLA-B *0801 and DQB1*02 with the European 8.1AH [20-23].

The role of DP molecules has yet to be resolved satisfactorily. The results favor DPB1*0301 and DPB1*0202 alleles as predisposing for T1DM [24]. Analysis of family-based data from the Human Biological Data Interchange (HBDI) repository and Italian studies, suggests the presence of a T1DM protective locus at or near DPB1*0101 [25]. It is hypothesized that the strongest candidates for increasing T1DM risk among DR3-DQB1*0201/DR4-DQB1*0302 individuals is of alleles of DP and DRB1*04 subtypes and, in particular, the absence of reportedly protective alleles DPB1*0402 and/or DRB1*0403 [26].

There are some studies on HLA-DP in different ethnic groups mainly about HLA-DPB1. In a study from Sudan, there were no significant differences between Sudanese patient and control groups in HLA-DPB1 frequencies [27]. Although, there was also no noticeable association between T1DM and HLA-DPB1 allele in Japanese [28]. From Indian T1DM patients, so far no studies have been reported on DP molecules.

Although different methods exist to characterize the polymorphisms in HLA genes, the $12^{\text {th }}$ International Histocompatibility Workshop suggested the Sequence Based Typing (SBT) methods [29]. Therefore, we conducted a hospital-based case-control study in the West Bengal region of India to find out the role of HLA- DRB1, DQ and DP gene polymorphisms in progress T1DM.

\section{Methods \\ Subjects}

In the present study 151 T1DM patients were recruited from six different hospitals - Calcutta Heart and Research Clinic; Endocrinology Department, Calcutta Medical College \& Hospital; Endocrinology Department, SSKM Hospital; Netaji Subhash Chandra Bose Cancer Research Institute; Rabindranath Research Institute of Cardiac Sciences; School of Tropical Medicine; from metropolitan Kolkata. The inclusion criteria considered for recruitment of cases were an onset of diabetes below 39 years of age, and presenting with or without acute ketosis with absolute insulin dependence, as shown by a deficient $\mathrm{C}$-peptide secretion i.e., a $\mathrm{C}$-peptide value less than $0.6(0.6-3.2) \mathrm{ng} / \mathrm{ml}$ and antibody positivity for Glutamic Acid Decarboxylase antigen(GADA) and, Insulinoma-Associated Protein-2 Antibodies (/IA-2/ICA512) [30]. Patients of at least one year duration were selected to exclude acute or "honeymoon" phases.

The sampled subjects speak Bengali. The samples represented in our study is mainly originated from

Table 1 List of primers used in the present study to amplify genes

\begin{tabular}{|c|c|c|c|c|}
\hline Designation & Chromosome location & Sequence & Annealing temperature & Product size(bp) \\
\hline \multirow[t]{2}{*}{ HLA-DPA $1^{*}$} & 6p21.3 (Exon2) & F-5' GCGGACCATGTGTGTCAACTTAT 3' & $55^{\circ} \mathrm{C}$ & 210 \\
\hline & & R-5' GCCTGAGTGTGGTTGGAACG 3' & & \\
\hline \multirow[t]{2}{*}{ HLA-DPB1* } & 6p21.3 (Exon2) & F-5' GAGAGTGGCGCCTCCGCTCAT 3' & $63^{\circ} \mathrm{C}$ & 327 \\
\hline & & R-5' GCCGGCCCAAAGCCCTCACTC 3' & & \\
\hline \multirow[t]{6}{*}{ HLA-DQA1 } & $6 \mathrm{p} 21.3^{\$}$ (Exon1) & F-5' CAAACTCTTCAGCTAGTAAC 3' & $58^{\circ} \mathrm{C}$ & 262 \\
\hline & & R-5' CATGCACTCACCCACAAT 3' & & \\
\hline & $6 \mathrm{p} 21.3^{*}($ Exon2) & F-5' ATGGTGTAAACTTGTACCAGT 3' & $55^{\circ} \mathrm{C}$ & 229 \\
\hline & & R-5' TTGGTAGCAGCGGTAGAGTTG 3' & & \\
\hline & $6 \mathrm{p} 21.3^{\$}$ (Exon3) & F-5' AGGTTCCTGAGGTCACAGTGTTT 3' & $54^{\circ} \mathrm{C}$ & 328 \\
\hline & & R-5' CTTGACAGACAAGAAAGCATC 3' & & \\
\hline \multirow[t]{2}{*}{ HLA-DQB1* } & 6p21.3(Exon2) & F-5' CATGTGCTACTTCACCAACGG 3' & $58^{\circ} \mathrm{C}$ & 211 \\
\hline & & R-5' CTGGTAGTTGTGTCTGCACAC 3' & & \\
\hline \multirow[t]{2}{*}{ HLA-DRB1* } & 6p21.3(Exon2) & F-5' CCCCACAGCACGTTTCTTG 3' & $60^{\circ} \mathrm{C}$ & 274 \\
\hline & & R-5' CCGCTGCACTGTGAAGCTCT 3' & & \\
\hline
\end{tabular}


districts-Kolkata, followed by South-24-Parganas, North24Parganas, Howrah, Hoogly, comprising a small geographical area and historically forming a cultural zone. West Bengal is the melting pot of Indo-Aryan, Austric, Dravidian, Tibeto-Burman and various other languages [31]. Some scholar stated that Bengal had many striking resemblance with the Dravidian culture [32], where as others suggested that Bengal as the meeting place of Aryan, non-Aryan, and Mongoloid races [33].

The controls used in the present study represent 151 healthy individuals without T1DM and T2DM in the family history, and matched for ethnicity, geography and socioeconomic status and higher age compared to cases. Blood samples $(3 \mathrm{ml})$ were collected into EDTA-coated vacutainers from both cases and controls with written informed consent. This research was approved by the Institutional Review Board of the Anthropological Survey of India as well as by the respective hospital's ethical committee.

\section{Genotyping}

DNA was isolated according to the standard protocol [34]. Genotyping of the following HLA genes HLADPA1, HLA-DPB1, HLA-DQA1, HLA-DQB1, and HLA-DRB1 was performed using PCR followed by sequencing. The primers used in the present study to amplify different regions of aforementioned genes are documented in Table 1. A total volume of $10 \mu \mathrm{l}$ was used for each PCR reaction which were carried out in an ABI Gene Amp PCR system 9700. The nucleotide

Table 2 Genotyping of SNPs in LD blocks

\begin{tabular}{|c|c|c|c|c|c|c|c|c|}
\hline & Genotype & & & MAF (\%) & HWE-p value & Dominant OR ( $p$ value) & Recessive OR ( $p$ value) & Allelic OR(p value) \\
\hline rs1047993 & $\pi$ & TC & CC & & & & & \\
\hline Cases & $2(1.45)$ & $26(18.84)$ & $110(79.79)$ & 10.9 & 0.745 & & & \\
\hline Control & $2(1.33)$ & $18(12.0)$ & $130(86.67)$ & 7.3 & 0.152 & $1.65(0.114)$ & $1.09(0.933)$ & $1.54(0.139)$ \\
\hline rs9272699 & AA & $A C$ & CC & & & & & \\
\hline Cases & $2(1.42)$ & $15(10.64)$ & $124(87.94)$ & 6.7 & 0.068 & & & \\
\hline Control & $0(0)$ & $15(10.14)$ & $133(89.86)$ & 5.1 & 0.516 & $1.22(0.603)$ & & $1.35(0.394)$ \\
\hline rs1048052 & CC & $A C$ & AA & & & & & \\
\hline Cases & $4(2.84)$ & $17(12.06)$ & $120(85.11)$ & 8.9 & 0.003 & & & \\
\hline Control & $0(0)$ & $17(11.49)$ & $131(88.51)$ & 5.7 & 0.459 & $1.35(0.392)$ & & $1.60(0.148)$ \\
\hline rs36219014 & $\pi$ & CT & $\mathrm{CC}$ & & & & & \\
\hline Cases & $0(0)$ & $17(12.06)$ & $124(87.94)$ & 6 & 0.446 & & & \\
\hline Control & $0(0)$ & $15(10.27)$ & 131 (89.73) & 5.1 & 0.513 & $120(0.631)$ & & $1.18(0.642)$ \\
\hline rs707962 & GG & GT & $\pi$ & & & & & \\
\hline Cases & $0(0)$ & $30(20.69)$ & 115 (79.31) & 10.3 & 0.165 & & & \\
\hline Control & $0(0)$ & $19(12.58)$ & $132(87.42)$ & 6.3 & 0.409 & $1.81(0.061)$ & & $1.72(0.074)$ \\
\hline rs2308911 & CC & CA & AA & & & & & \\
\hline Cases & 15 (10.95) & $66(48.18)$ & $56(40.88)$ & 35 & 0.495 & & & \\
\hline Control & $14(10.37)$ & 61 (45.19) & $60(44.44)$ & 33 & 0.795 & $1.16(0.552)$ & $1.06(0.877)$ & $1.10(0.610)$ \\
\hline rs2308912 & $\mathrm{AA}$ & AT & $\pi$ & & & & & \\
\hline Cases & $14(10.0)$ & $64(45.71)$ & $62(44.29)$ & 32.9 & 0.669 & & & \\
\hline Control & $12(9.09)$ & $61(46.21)$ & $59(44.70)$ & 32.2 & 0.502 & $1.02(0.946)$ & $1.11(0.799)$ & $1.03(0.870)$ \\
\hline rs707949 & $\mathrm{CC}$ & $\mathrm{CT}$ & $\pi$ & & & & & \\
\hline Cases & $0(0)$ & 30 (20.69) & 115 (79.31) & 10.3 & 0.165 & & & \\
\hline Control & $0(0)$ & $20(13.25)$ & 131 (86.75) & 6.6 & 0.384 & $1.71(0.087)$ & & $1.63(0.104)$ \\
\hline rs7990 & AA & $A C$ & $\mathrm{CC}$ & & & & & \\
\hline Cases & $0(0)$ & $14(9.72)$ & $130(90.28)$ & 4.9 & 0.549 & & & \\
\hline Control & $0(0)$ & $31(20.53)$ & $120(79.47)$ & 10.3 & 0.160 & $0.42(0.009)$ & & $0.45(0.013)$ \\
\hline rs707963 & GG & GT & $\pi$ & & & & & \\
\hline Cases & $0(0)$ & $28(19.31)$ & 117 (80.69) & 9.7 & 0.198 & & & \\
\hline Control & $0(0)$ & $18(11.92)$ & $133(88.08)$ & 6 & 0.436 & $1.77(0.079)$ & & $1.69(0.093)$ \\
\hline
\end{tabular}




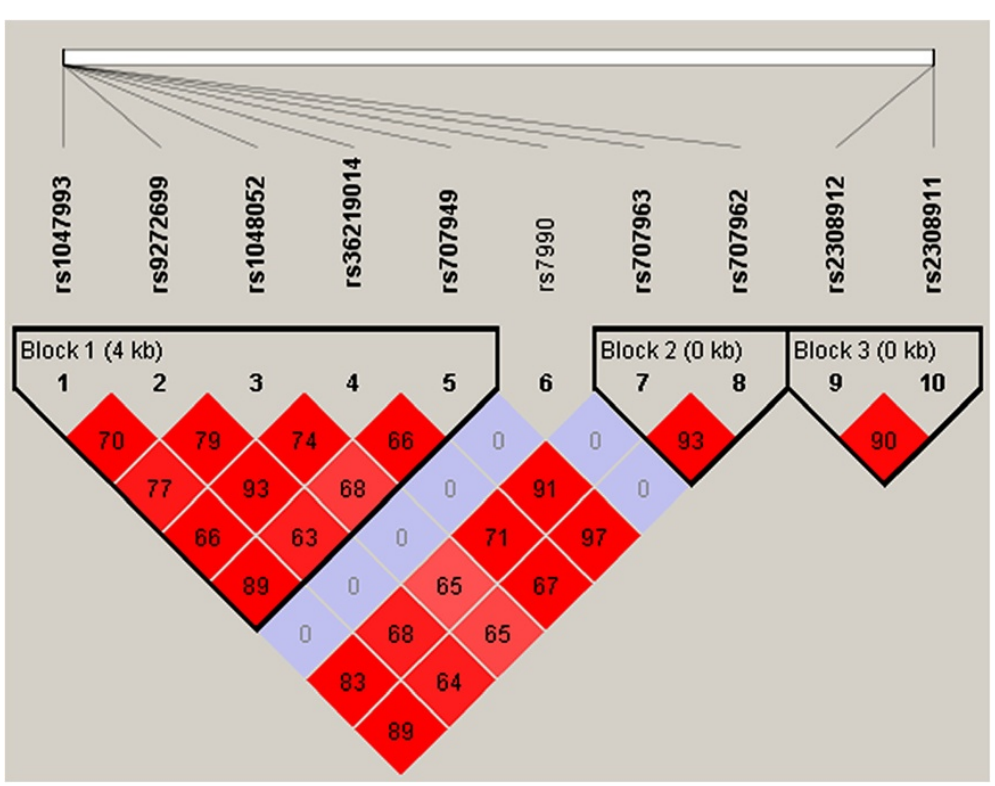

Figure 1 Pair-wise linkage disequilibrium between the ten SNP markers in HLA-DQA1-DPA1 genes.

sequences of the PCR products were determined by direct sequencing using di-deoxy chain terminator cycle sequencing protocol through 3730 DNA Analyzer (BigDye V3.1, Applied Biosystems; Foster City, CA, USA). Sequencing was carried out with both the forward and the reverse directions. Problems in genotype assignment for certain samples on the DRB1 and the DQB1 loci that aroused because of the genotypic ambiguity were surmounted by following the guidelines of the American Society for Histocompatibility and Immunogenetics [35].

\section{Statistical analysis}

All nucleotide changes detected by using SeqScape software v 2.5 (Applied Biosystems) and with the wild gene using pair-wise BLAST [36]. Class II HLA genes are more polymorphic compared to other genes and thus the editing was much more complicated for Class II HLA. Allele and genotype frequencies of the SNP data compared between T1DM patients and healthy control groups. Statistical analysis for HWE was performed by Plink v 1.07 software [37]. Evaluation of the genotype or allele frequencies of cases and controls was carried out by calculating the odds ratios (OR) with $95 \%$ of confidence intervals (CI). A $P$ value less than 0.05 were considered as statistically significant. Haplotype frequencies and linkage disequilibrium estimated by using Haploview v 4.1, which measures D' and $\mathrm{r}^{2}$ between each pair of SNPs and to define haploblocks [38].

\section{Results and discussions}

Exonic 331 SNPs in the Class II HLA- DP-DQ-DRB1, identified and tested for HWE by Plink software. Only ten SNPs found to be in equilibrium for control, only these 10 SNPs were used in further analysis. Of the 10 SNPs, eight were from HLA-DQA1 and the remaining were from HLA-DPA1 (rs2308911, rs2308912) (Table 2). Allele and genotype frequencies of all the SNPs for both cases and controls were presented in Table 2. Except for rs7990 ( $p=0.009$ ), there were no significant differences in genotype or allele frequencies of these SNPs between controls and T1DM cases (Table 2). Thus, after all prune and excluding from 331 SNPs, rs7990 SNPs of HLADQA1 shows a negative association with T1DM. The pairwise LD values (D' and r2) among studied SNPs were provided in Figure 1 and in Table 3. Linkage

Table 3 HLA-DQA1 and-DPA1 gene haplotypes and T1DM

\begin{tabular}{lllll}
\hline \multicolumn{5}{l}{ rs1047993, rs9272699, rs1048052, rs36219014 and rs707949 } \\
\hline Haplotype & Control & Case & $\boldsymbol{X}^{\mathbf{2}}$ & $\boldsymbol{p}$-value \\
\hline CCACT & $\mathbf{0 . 8 8 3}$ & $\mathbf{0 . 9 3 9}$ & $\mathbf{5 . 6 7 2}$ & $\mathbf{0 . 0 1 7}$ \\
TACTC & 0.060 & 0.051 & 0.255 & 0.614 \\
TCACC & $\mathbf{0 . 0 2 8}$ & $\mathbf{0 . 0 0 3}$ & $\mathbf{5 . 8 7 5}$ & $\mathbf{0 . 0 1 5}$ \\
rs707963 and rs707962 & & & \\
Haplotype & Control & Case & $X^{2}$ & p-value \\
TT & 0.897 & 0.937 & 3.202 & 0.074 \\
GG & 0.097 & 0.060 & 2.818 & 0.093 \\
rs2308911 and rs2308912 & & & \\
Haplotype & Control & Case & $x^{2}$ & $p$-value \\
TA & 0.650 & 0.663 & 0.107 & 0.744 \\
AC & 0.336 & 0.307 & 0.501 & 0.479 \\
TC & 0.015 & 0.022 & 0.436 & 0.509 \\
\hline X & & & &
\end{tabular}


disequilibrium analysis had revealed strong LD and formed 3 haplotype blocks, suggested that haplotype study might be useful. Haplotype-phenotype association analysis using SNPs that were located in the LD blocks in block 1 (rs1047993 and rs707949) showed association with T1DM (Table 3).

\section{Conclusions}

The complex nature of the HLA region on chromosome 6 p21.31, with the high LD between genes, has made it enormously difficult to explicate the effect of individual genes for the risk of developing T1DM. The studies suggested that HLA Class II DRB1-DQB1 contribute to T1DM susceptibility. Calculating for the influence of class II DR-DQ haplotype and genotype effects, a role in T1DM has been shown of additional HLA Class II DPB1[16].

New studies by sequencing has yielded many SNPs that include thirteen SNPs from class III alleles, which showed evidence of an effect on T1DM risk, although some of the SNPs are in tight LD with each other. The strongest association within class III markers was with rs2395106 that maps $5^{\prime}$ to the NOTCH4 gene and the second association was with rs707915 mapping to the MSH5 gene, in a block of six markers significantly associated with T1DM after adjusting for LD with DR-DQ [39].

A widespread SNP analysis of the extended MHC in 237 families with Type 1A from the U.S. and 1,240 families from the T1DGC was conducted and showed an association with Type $1 \mathrm{~A}$ diabetes ( $\mathrm{rs} 1233478, \mathrm{p}=1.6 \mathrm{x}$ $10^{-23}$ ), in the UBD/MAS1L region, telomeric of the classic MHC [40]. Another study from T1DGC on HLA markers showed 296 significant SNPs in a narrow genomic region, some of these markers are close to one another and in strong LD. Therefore, although the SNPs that stand for independent signals without LD, some high-LD markers can produce correlated associations in the study. However, high-LD and long haplotype blocks will also deter fine mapping precisely. This study also shows that SNPs with the smallest $P$ values is from the HLA-DR and -DQ region which confers the major genetic risks for T1DM [41].

Most of these recent studies have used data produced by the T1DGC and therefore are from Caucasoid population, and they conducted the most detailed investigation of the HLA complex in disease, characterizing over 3,000 SNPs, and independently tested all previously reported T1DM susceptibility genes [42,43].

In this study we used a sequencing SNP approach to characterize the polymorphisms in HLA genes. We carried out sequencing in 151 cases and 151 normal healthy participants, followed by statistical analysis of the SNPs. Out of 331 exonic SNPs identified, only 10 is following HWE. Thus, after all pruning and excluding from 331 SNPs, and rs7990 SNP of HLA-DQA1 showed significant protection from T1DM. Linkage disequilibrium analysis revealed 3 haplotype blocks and further haplotype-phenotype association analysis did show association between haplotypes in block 1 (rs1047993 and rs707949) and T1DM.

We have also done the study based on alleles and the findings are similar like the DQA1*0103 allele is a novel allele with a significant association with the protection from T1DM in Eastern Indian Bengali population from India [44]. To our knowledge, this study is the first of a kind which has tried to check the HLA association with T1DM by SNPs analysis in India. As said before most of these recent studies have used data formed by the T1DGC, and they use refined statistical methods to control for the complexity of the HLA region because of the extended LD and polymorphic loci. Still the deviation of some results suggests the difficulty of examine the independent genetic contribution of genes in this region to the risk of developing T1DM. It is most likely that more SNPs with individual but smaller or rarer effects on diabetes risk can be identified in this region. However, to find these SNPs new approaches for analyzing genetic association data are needed. In addition, large numbers of subjects may help to give more robust and confident association with T1DM.

\section{Competing interest}

There are no conflicts of interest.

\section{Authors' contributions}

OR carried out the molecular genetic studies, participated in the sequence alignment, did the statistical calculation and drafted the manuscript. BS corrected the manuscript. BLVKS conducted the Thesia calculation. VP suggested the project GS suggested the project SC provided with the samples PR provided with the samples VRR guided the total project and corrected the MS. All authors read and approved the final manuscript.

\section{Acknowledgements}

This research work is supported by the grant from Anthropological Survey of India, Ministry of Culture, Government of India. We are thankful to the T1DM patients and control subjects for voluntarily taking part in this research work and donating their blood samples. Mr. Nupur Gowswami has helped in the collection of blood samples from the OPD of CMC Hospital.

\section{Author details}

${ }^{1}$ Anthropological Survey of India, Kolkata, West Bengal 700016, India. ${ }^{2}$ Department of Biomedical Sciences, Sri Ramachandra University, Chennai 600 116, India. ${ }^{3}$ Department of Human Genetics, Andhra University, Visakhapatnam 530003, India. ${ }^{4}$ Endocrinology Department, SSKM Hospital, Kolkata, India. ${ }^{5}$ Calcutta Medical College \& Hospital, Kolkata, India. ${ }^{6}$ Department of Anthropology, University of Delhi, North Campus, Delhi 110 007 , India.

Received: 7 January 2013 Accepted: 25 February 2013

Published: 27 February 2013

\section{References}

1. Atkinson M, Maclaren $\mathrm{N}$ : The pathogenesis of insulin dependent diabetes. N Engl J Med 1994, 331:1428-1436.

2. Imagawa A, Hanafusa T, Miyagawa J, Matsuzawa Y: A novel subtype of type 1 diabetes mellitus characterized by a rapid onset and an absence of diabetes-related antibodies. N Engl J Med 2000, 342:301-307. 
3. Kuzuya T, Nakagawa S, Satoh J, Nanjo K, Sasaki A, Committee of the Japan Diabetes Society on the diagnostic criteria of diabetes mellitus: Report of the committee on the classification and diagnostic criteria of diabetes mellitus. Diabetes Res Clin Pract 2002, 55:65-85.

4. Puavilai G, Chanprasertyotin S, Sriphrapradaeng A: Diagnostic criteria for diabetes mellitus and other categories of glucose intolerance: 1997 criteria by the Expert Committee on the Diagnosis and Classification of Diabetes Mellitus (ADA);1998 WHO consultation criteria; and,WHO criteria:World Health Organization. Diabetes Res Clin Pract 1985, 1999(44):21-26.

5. Ramachandran A, Snehalatha C, Abdul Khader OM, Joseph TA, Viswanathan M: Prevalence of childhood diabetes in urban population in South India. Diabetes Res Clin Pract 1992, 17:227-231.

6. Ramachandran A, Snehalatha C, Krishnaswamy CV, Madras IDDM, Registry Group-Madras: Incidence of IDDM in children in urban population in southern India. Madras IDDM Registry Group Madras, South India. Diabetes Res Clin Pract 1996, 34:79-82

7. Kalra S, Kalra B, Sharma A: Prevalence of type 1 diabetes mellitus in karnal district, Haryana state, India. Diabetol Metab Syndr 2010, 2:1-3.

8. Motzo C, Contu D, Cordell HJ, Lampis R, Congia M, Marrosu MG, Todd JA, Cucca F: Heterogeneity in the magnitude of the insulin gene effect on HLA risk in type 1 diabetes. Diabetes 2004, 53:3286-3291.

9. Cudworth A, Woodrow J: Evidence for HLA-linked genes in "juvenile" diabetes mellitus. BMJ 1975, 3:133-135.

10. Kelly M, Rayner ML, Mijovic CH, Barnett AH: Molecular aspects of type 1diabetes. Mol Pathol 2003, 56:1-10.

11. IMGT/HLA Database.. http://www.ebi.ac.uk/imgt/hla/stats.html.

12. Murao S, Makino H, Kaino Y, Konoue E, Ohashi J, Kida K, Fujii Y, Shimizu I, Kawasaki E, Fujiyama $M$, et al: Differences in the contribution of HLA-DR and -DQ haplotypes to susceptibility to adult and childhood-onset type 1 diabetes in Japanese patients. Diabetes 2004, 53:2684-2690.

13. Weitkamp L: HLA and disease: predictions for HLA haplotype sharing in families. Am J Hum Genet 1981, 33:776-784.

14. Wolf $E$, Spencer KM, Cudworth AG: The genetic susceptibility to type 1 (insulin-dependent) diabetes: analysis of HLA-DR association. Diabetologia 1983, 24:224-230.

15. Florez J, Hirschhorn J, Altshuler D: The inherited basis of diabetes mellitus: implications for the genetic analysis of complex traits. Annu Rev Genomics Hum Genet 2003, 4:257-291.

16. Varney M, Valdes AM, Carlson JA, Noble J, Tait BD, Bonella P, et al: HLA DPA1, DPB1 alleles and haplotypes contribute to the risk associated with type 1 diabetes: analysis of the type 1 diabetes genetics consortium families. Diabetes 2010, 59:2055-2062.

17. Rajalingam $R, G e P$, Reed $E$ : A sequencing-based typing method for HLADQA1 alleles. Hum Immunol 2004, 65:373-379.

18. Rani R, Sood A, Goswami R: Molecular basis of predisposition to develop type 1 diabetes mellitus in North Indians. Tissue Antigens 2004, 64:145-155.

19. Shtauvere A, Kanungo A, Samal KC, Tripathi BB, Sanjeevi CB: Association of HLA class II alleles with different subgroups of diabetes mellitus in Eastern India identify different associations with IDDM and malnutritionrelated diabetes. Tissue Antigens 1999, 54:83-87.

20. Mehra N, Jaini R, Rajalingam R, Balamurugan A, Kaur G: Molecular diversity in Asian Indians: predominance of $a^{*} 0211$. Tissue Antigens 2001, 57:502-507.

21. Rani R, Sood A, Lazaro AM, Stastny P: Associations of MHC class II alleles with insulin-dependent diabetes mellitus (IDDM) in patients from North India. Hum Immunol 1999, 60:524-531.

22. Torn C, Gupta M, Zake NL, Sanjeevi CB, Landin-Olsson M: Heterozygosity for MICA50/MICA51 and HLA-DR3-DQ2/DR4-DQ8 are independent genetic risk factors for latent autoimmune diabetes mellitus in adults. Hum Immunol 2003, 64:902-909.

23. Witt C, Price P, Kaur G, Cheong K, Kanga U, Sayer D, Christiansen F, Mehra NK: Common HLA-B8-DR3 haplotype in Northern India is different from that found in Europe. Tissue Antigens 2002, 60:474-480.

24. Noble J, Valdes AM, Thomson G, Erlich HA: The HLA class II locus DPB1 can influence susceptibility to type 1 diabetes. Diabetes 2000, 49:121-125.

25. Noble J, Martin A, Valdes AM, Lane JA, Galgani A, Petrone A, et al: Type 1 diabetes risk for human leukocyte antigen (HLA)-DR3 haplotypes depends on genotypic context: association of DPB1 and HLA class I loci among DR3- and DR4-matched Italian patients and controls. Hum Immunol 2008, 69:291-300.

26. Bascha I, Aly TA, Babu SR, Fernando MS, Yu L, Miao D, Barriga KJ, Norris JM Noble JA, Erlich HA, et al: HLA-DPB1*0402 protects against type $1 \mathrm{~A}$ diabetes autoimmunity in the highest risk DR3-DQB1*0201/DR4DQB1*0302 DAISY population. Diabetes 2007, 56:2405-2409.

27. Magzoub M, Stephens HA, Sachs JA, Biro PA, Cutbush S, Wu Z, Bottazzo GF: HLA-DP polymorphism in Sudanese controls and patients with insulindependent diabetes mellitus. Tissue Antigens 1992, 40:64-68.

28. Yamagata $K$, Hanafusa T, Nakajima $H$, Sada M, Amemiya $H$, Tomita $K$, et al: HLA-DP and susceptibility to insulin-dependent diabetes mellitus in Japanese. Tissue Antigens 1991, 38:107-110.

29. Cordovado S, Simone AE, Mueller PW: High-resolution sequence-based typing strategy for HLA-DQA1 using SSP-PCR and subsequent genotyping analysis with novel spreadsheet program. Tissue Antigen 2001, 58:308-314.

30. Raha O, Sarkar BN, Bhaskar LVKS, Veerraju P, Chowdhury S, Mukhopadhyay S, Biswas T, Rao V: Insulin (INS) promoter VNTR polymorphisms: interactions and the association with type 1 diabetes mellitus in Bengali speaking patients of Eastern India. Diabetoligia Croatica 2011, 40:99-106.

31. Chattopadhay S: The origin and development of Bengali languages. Calcutta: George and Unwin Ltd.; 1926.

32. Singh K: Linguistic traits across language boundaries. Kolkata: Anthropological Survey of India; 1990

33. Majumdar D, Rao C: Race elements in Bengal: aquanititative study Indian statistical series-3. Calcutta: Asia Publishing House; 1960

34. Sambrook J, Russel DW: Molecular cloning: A laboratory manual. 3rd edition. Cold Spring Harbor; New York: Cold Spring Harbor Laboratory Press; 2001.

35. Cano P, Klitz W, Mack SJ, Maiers M, Marsh SGE, Noreen H, Reed EF, Senitzer D, Setterholm M, Smith A, Fernández-Viña M: Common and well-documented HLA alleles: report of the ad-hoc committee of the American society for histocompatiblity and immunogenetics. Hum Immuno/ 2007, 68:392-417.

36. Tatusova T, Madden T: BLAST 2 sequences a new tool for comparing protein and nucleotide sequences. FEMS Microbiol Lett 1999, 174:247-250.

37. Purcell S, Neale B, Todd-Brown K, Thomas L, Ferreira MAR, Bender D, Maller J, Sklar P, de Bakker PI, Daly MJ, Sham PC: PLINK: a toolset for whole-genome association and population-based linkage analysis. Am J Hum Genet 2007, 81:559-575.

38. Barrett J, Fry B, Maller J, Daly MJ: Haploview: analysis and visualization of LD and haplotype maps. Bioinformatics 2005, 21:263-265.

39. Valdes A, Thomson G: Type 1 Diabetes Genetics Consortium: Several loci in the HLA class III region are associated with T1D risk after adjusting for DRB1-DQB1. Diabetes Obes Metab 2009, Suppl 1:46-52.

40. Aly $T$, Baschal EE, Jahromi MM, Fernando MS, Babu SR, Fingerlin TE, Kretowski A, Erlich HA, Fain PR, Rewers MJ, Eisenbarth GS: Analysis of single nucleotide polymorphisms identifies major type $1 \mathrm{~A}$ diabetes locus telomeric of the major histocompatibility complex. Diabetes 2008 , 57:770-776.

41. He C, Hamon S, Li D, Barral-Rodriguez SJO, Diabetes Genetics Consortium: MHC fine mapping of human type 1 diabetes using the T1DGC data. Diabetes Obes Metab 2009, 11:53-59.

42. Barrett JC, Clayton DG, Concannon P, Akolkar B, Cooper JD, Erlich HA, Julier C, Morahan G, Nerup J, Nierras C, et al: Genome-wide association study and meta-analysis find that over 40 loci affect risk of type 1 diabetes. Nat Genet 2009, 41:703-707.

43. Morahan G, Mehta M, James I, Chen WM, Akolkar B, Erlich HA, Hilner JE, Julier C, Nerup J, Nierras C, et al: Tests for genetic interactions in type 1 diabetes: linkage and stratification analyses of 4,422 affected sib-pairs. Diabetes 2011, 60:1030-1040.

44. Raha O, Sarkar B, Veerraju P, Sudhakar G, Raychaudhuri P, Mukhopadhyay S, Rao V: Role of HLA class II loci polymorphism in the manifestation of type 1 diabetes in a Bengali Indian patient population. Genet Test $\mathrm{Mol}$ Biomarkers 2013, 17:52-61.

doi:10.1186/1423-0127-20-12

Cite this article as: Raha et al:: HLA class II SNP interactions and the association with type 1 diabetes mellitus in Bengali speaking patients of Eastern India. Journal of Biomedical Science 2013 20:12. 